\title{
Open-boundary modal analysis: Interpolation, extrapolation, and filtering
}

\author{
F. Lekien ${ }^{1}$ and C. Coulliette \\ Control and Dynamical Systems, California Institute of Technology, Pasadena, California, USA \\ R. Bank \\ Department of Mathematics, University of California, San Diego, La Jolla, California, USA
}

\section{J. Marsden}

Control and Dynamical Systems, California Institute of Technology, Pasadena, California, USA

Received 12 February 2004; revised 27 June 2004; accepted 26 July 2004; published 4 December 2004.

[1] Increasingly accurate remote sensing techniques are available today, and methods such as modal analysis are used to transform, interpolate, and regularize the measured velocity fields. Until recently, the modes used did not incorporate flow across an open boundary of the domain. Open boundaries are an important concept when the domain is not completely closed by a shoreline. Previous modal analysis methods, such as those of Lipphardt et al. (2000), project the data onto closed-boundary modes, and then add a zero-order mode to simulate flow across the boundary. Chu et al. (2003) propose an alternative where the modes are constrained by a prescribed boundary condition. These methods require an a priori knowledge of the normal velocity at the open boundary. This flux must be extrapolated from the data or extracted from a numerical model of a larger-scale domain, increasing the complexity of the operation. In addition, such methods make it difficult to add a threshold on the length scale of open-boundary processes. Moreover, the boundary condition changes in time, and the computation of all or some modes must be done at each time step. Hence real-time applications, where robustness and efficiency are key factors, were hardly practical. We present an improved procedure in which we add scalable boundary modes to the set of eigenfunctions. The end result of open-boundary modal analysis (OMA) is a set of time and data independent eigenfunctions that can be used to interpolate, extrapolate and filter flows on an arbitrary domain with or without flow through segments of the boundary. The modes depend only on the geometry and do not change in time. INDEX TERMS: 3210 Mathematical Geophysics: Modeling; 3220 Mathematical Geophysics: Nonlinear dynamics; 3299 Mathematical Geophysics: General or miscellaneous; 4275 Oceanography: General: Remote sensing and electromagnetic processes (0689); KEYWORDS: modal analysis, interpolation, filtering

Citation: Lekien, F., C. Coulliette, R. Bank, and J. Marsden (2004), Open-boundary modal analysis: Interpolation, extrapolation, and filtering, J. Geophys. Res., 109, C12004, doi:10.1029/2004JC002323.

\section{Introduction}

[2] Dynamical systems theory provides tools to extract alleyways and barriers to Lagrangian transport in fluid flows. Recent works show that the detection and extraction of the Lagrangian structures governing transport and mixing in coastal and oceanographic flow can be used to optimize the path of drifters and underwater gliders, or to minimize the impact of coastal pollution [see, e.g., Coulliette and Wiggins, 2001; Lekien et al., 2003]. These methods assume

\footnotetext{
${ }^{1}$ Now at Department of Mechanical and Aerospace Engineering, Princeton University, Princeton, New Jersey, USA.

Copyright 2004 by the American Geophysical Union. 0148-0227/04/2004JC002323\$09.00
}

that the motion of a particle of water or a passive drifter in the ocean is given by

$$
\dot{\bar{x}}=\bar{u}(\bar{x}, t)
$$

where $\bar{x}$ is the position (longitude and latitude) of the particle and $\bar{u}$ is the time-dependent two-dimensional velocity field. The velocity field $\bar{u}$ is typically approximated by an analytical equation or replaced by a numerical simulation of a large-scale ocean model. However, in the last few years, technological advances in techniques such as high-frequency radiometry provided accurate measurements of the velocity vectors in certain coastal areas [Paduan and Cook, 1997], and our goal is to provide a recipe to replace $\bar{u}$ in equation (1) with experimental data. These data cannot be 
used directly as a dynamical system, unlike a smooth ordinary differential equation (ODE) that can be integrated. There are usually gaps due to the radar range and wind conditions [Paduan and Cook, 1997]. As a result, oceanographers are in need of a way to extrapolate, interpolate, and filter such data. The resulting smoothed velocity field is often called a "nowcast" in reference to the much more common forecasting operation. Nowcasting does not involve the extrapolation of the velocity into the future. It uses available data to determine the velocity everywhere in space at the same time that the data were collected.

[3] Eigenvalue problems provide basis functions over a domain, and this technique has been used in fields such as electromagnetism or quantum mechanics. Recent works [Eremeev et al., 1992a, 1992b, 1995a, 1995b; Lipphardt et al., 2000] adapt these techniques to geophysical flows. In the case of a coastal oceanographic process, the boundary conditions may not always be natural. The boundary of some regions of interest does not correspond exactly to the shoreline (such as in the work by Lipphardt et al. [2000]). In most cases, the region of interest is closed by a segment of shoreline on one side and an artificial boundary in the sea, resulting from the limited range of action of the radarantenna system or the boundary of a coastal ocean model. In such cases, one needs to take into account the fact there can be a non-zero normal flux through certain portions of the boundary. This flux can be positive or negative across different portions of the open boundary.

[4] Lipphardt et al. [2000] propose to append a zeroorder mode satisfying a prescribed boundary condition at the open boundary. The space of all available homogeneous velocity fields is translated to force each solution to match the prescribed boundary condition. This is satisfactory only if the exact boundary condition is known. However, the flux through the boundary must be extrapolated from the data or, as Lipphardt et al. [2000] suggest, extracted from a numerical model covering a larger domain. Chu et al. [2003] showed later that the solution can be extremely sensitive to the method used to extrapolate the data or numerical errors in the modeled open-boundary flow. In addition, this procedure requires the numerical computation of the zeroorder mode at each time step. This complicates its use and decreases the efficiency in real-time applications where computational time and human intervention are extremely costly.

[5] An alternative method is presented by Chu et al. [2003], where the modes from Lipphardt et al. [2000] are modified in such a way that they all satisfy a prescribed approximation of the open-boundary condition. Chu et al. [2003] also analyze and propose several methods to extrapolate the boundary conditions from the data. In this case, all the modes have to be computed at each time, creating a high computational load. The high demand in computation time makes the approach hardly practical for real-time applications. In addition, Chu et al. [2003] noticed the extreme sensitivity of the solutions on the prescribed boundary condition. Although they find a satisfactory method to approximate the flux through the boundary in their particular example, avoiding the necessary extrapolation of the boundary condition is a key element to improving the quality of the nowcast and decreasing the computational complexity of the operation.

[6] In this paper, we present a complete functional basis that can be used to project the flow on modes allowing flux through selected segments of the boundary. This method, called open-boundary modal analysis (i.e., OMA), is somewhat similar to, and builds on the method introduced by, Lipphardt et al. [2000]. However, there is a fundamental difference in that there is the addition of an infinite (possibly truncated) sequence of boundary modes, which allow adequate degrees of freedom to project the experimental data onto open-boundaries. The space of all available velocity fields is greatly increased. However, all the modes are data and time independent. They depend only on the geometry of the boundary. As a result, they can be computed once and stored for all future use. This allows for fast and robust nowcasts during real-time experiments. In addition, the larger space allows us to avoid the complicated operation of extrapolating open-boundary flux or making any assumptions about normal or tangential flow along the open boundary as other methods require. In our method, the projection of the data on the modes determines the optimal open-boundary flow. Another advantage (that we thank an anonymous referee for indicating) is that the approach we describe allows us to match boundary scales with the interior scales.

[7] Unlike other methods, the approach that we highlight renders the eigenfunctions on an unstructured triangular grid with a general boundary. For example, Lipphardt et al. [2000] use a staircase approximation of the actual coastline, where the approximate boundary is composed entirely of segments oriented at $0^{\circ}, 90^{\circ}, 180^{\circ}$, and $270^{\circ}$. There are three major reasons why an unstructured triangulation is superior to a normal grid with a staircase boundary approximation. First, a staircase boundary is a less accurate representation of a coastline geometry than the unstructured triangulation we use, unless an extremely fine staircase boundary is used, which is computationally intractable because it would require making the entire domain an equally fine mesh. In this case, not only would the computational time be prohibitive, but round-off error becomes a limiting factor.

[8] Second, at each corner vertex along a staircase boundary, the tangential velocity must be set to zero in order to satisfy the no-penetration condition along the coastline. Therefore it is not possible to have a boundary condition along a staircase coastline that is both free slip (or quasi-free slip) and no penetration. The unstructured triangulation used by OMA avoids this problem related to staircase boundaries. When computing the solutions on the unstructured mesh, natural boundary conditions can be imposed in a weak sense, i.e., under integrals. The integral on the boundary is decomposed as the sum of integrals on the individual edges of the boundary, so point values at the corners of the boundary do not need to be even defined. The points of $C^{1}$ discontinuity on the boundary are a set of measure zero. The integrals are approximated using 2 or 3 point Gauss quadrature rules, so the quadrature points do not include the vertices. The resulting flow can satisfy a free slip boundary condition while preventing penetration of the coastline by the particles. 
[9] Third, the numerical error in calculating the mode shapes is held constant across the domain and boundary by varying the size of the triangles in the unstructured approach. In comparison, a normal grid with constant-size elements results in a numerical error that varies in magnitude substantially across the domain and boundary [see, e.g., Lipphardt et al., 2000; Chu et al., 2003]. Thus the unstructured triangulation is more efficient, resulting in a much higher accuracy to computational time ratio.

\section{Stream Function and Relative Vorticity}

\subsection{State Equation Inside the Domain}

[10] The nowcast velocity will be denoted by a function $\bar{u}$ (with two components) on a compact subset $\Omega$ of a smooth two-dimensional manifold. In section 6 , we give an analytical example where $\Omega$ is the unit square. Section 9 focuses on a small region around Monterey Bay, California. In both cases, $\Omega \subset \mathbb{R}^{2}$. However, the mode equations derived in this paper are not dependent on the coordinate system and can be computed on any two-dimensional smooth manifold. The Hodge decomposition [Eiseman and Stone, 1973] states that $\bar{u}$ can always be written as the sum

$$
\bar{u}=\bar{u}_{\psi}+\bar{u}_{\phi}
$$

where

$$
\bar{u}_{\psi}=\nabla \times \psi \bar{k}
$$

is divergence-free and

$$
\bar{u}_{\phi}=\nabla \phi
$$

is irrotational. We recall how this proceeds: Combining equations (3) and (4) with equation (2) gives

$$
\bar{u}=\nabla \times(\psi \bar{k})+\nabla \phi
$$

where $\bar{k}$ is the unit vector orthogonal to the domain, pointing upwards. In the above equation, $\nabla \times$ applies to a vector oriented in the $\bar{k}$ direction. The result is a vector lying in the plane (i.e., the component along $\bar{k}$ vanishes). We consider this vector as a two-dimensional vector. Applying $\nabla \cdot$ and $\nabla \times$ to equation (5) gives

$$
\Delta \phi=\nabla \cdot \bar{u}
$$

and

$$
\begin{aligned}
\nabla \times \bar{u} & =\nabla \times \nabla \times(\psi \bar{k}) \\
& =-\Delta(\psi \bar{k})=-\bar{k} \Delta \psi,
\end{aligned}
$$

so

$$
\Delta \psi=-\bar{k} \cdot(\nabla \times \bar{u})
$$

where $\nabla \times \bar{u}$ is the curl of the velocity in the plane of interest. Note that $\nabla \times \bar{u}$ is often represented as a vector oriented along $\bar{k}$, hence its projection on $\bar{k}$ in equation (7).

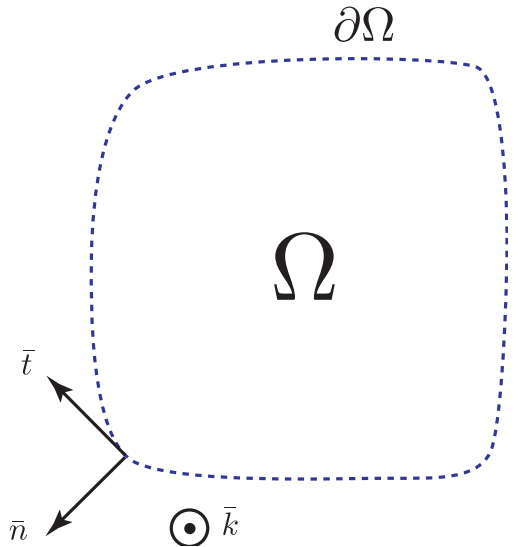

Figure 1. Domain $\Omega$ and its boundary $\partial \Omega$. The unit vector $\bar{k}$ is orthogonal to the ocean surface and points up to the reader. The vector $\bar{n}$ is the unit vector normal to the boundary $\partial \Omega$. The tangent vector $\bar{t}$ is given by $\bar{t}=\bar{n} \times \bar{k}$.

In the next section, we discuss the boundary conditions for the scalar function $\phi$ and $\psi$ defined by equations (6) and (7). Owing to the presence of segments of open boundary, the boundary conditions for these scalar functions are not completely defined. The objective of this paper is to investigate the influence of the boundary conditions applied to $\bar{u}$ on the spaces spanned by each scalar function $\phi$ and $\psi$. The basis (i.e., complete sequences) of these two sets provides a corresponding basis of the space of all available velocities.

\subsection{Boundary Conditions}

[11] We let $\partial \Omega$ denote the boundary of the compact domain $\Omega$. The unit vector normal to the boundary $\partial \Omega$ and pointing outside the domain is denoted $\bar{n}$ (Figure 1). The unit tangent vector is $\bar{t}=\bar{n} \times \bar{k}$, as indicated in the figure. We assume that the boundary $\partial \Omega$ is continuous and piecewise $C^{1}$ (that is, for all but a finite number of points on the boundary, there exists an open set $U_{x}$ containing $x$ such that $U_{x} \cap \partial \Omega$ is the graph of a $C^{1}$ continuous function). Taking the dot product of equation (5) with $\bar{t}$ and $\bar{n}$ gives

$$
\begin{aligned}
\bar{u} \cdot \bar{t} & =\bar{t} \cdot(\nabla \times \psi \bar{k})+\bar{t} \cdot \nabla \phi \\
& =\bar{t} \cdot \nabla \phi+\bar{n} \cdot \nabla \psi
\end{aligned}
$$

and

$$
\begin{aligned}
\bar{u} \cdot \bar{n} & =\bar{n} \cdot(\nabla \times \psi \bar{k})+\bar{n} \cdot \nabla \phi \\
& =\bar{n} \cdot \nabla \phi-\bar{t} \cdot \nabla \psi,
\end{aligned}
$$

which can be used to establish the boundary conditions for the scalar functions $\phi$ and $\psi$.

[12] We assume that the boundary $\partial \Omega$ is made of the union of two different boundary pieces,

$$
\left\{\begin{array}{l}
\partial \Omega=\partial \Omega_{0} \cup \partial \Omega_{1} \\
\partial \Omega_{0} \cap \partial \Omega_{1}=\emptyset,
\end{array}\right.
$$




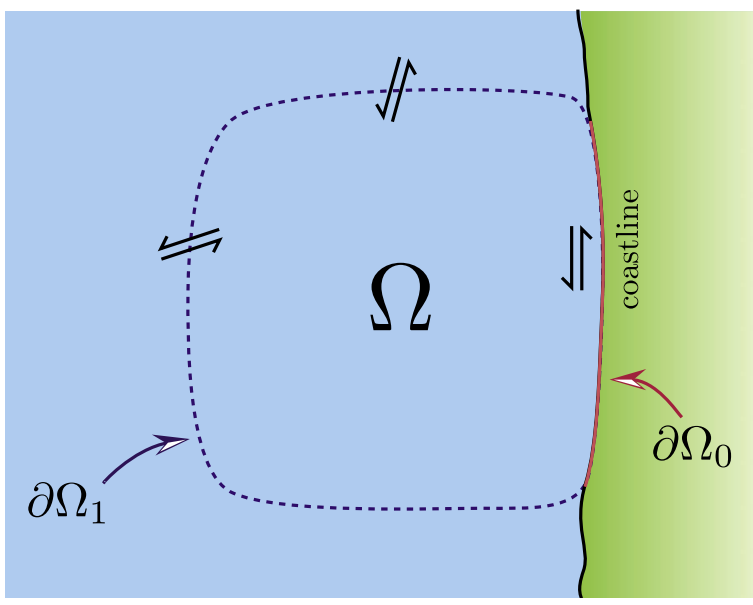

Figure 2. Domain $\Omega$ and its boundary $\partial \Omega=\partial \Omega_{0} \cup \partial \Omega_{1}$. The portion $\partial \Omega_{0}$ is made of solid ground and has no normal flow, while $\partial \Omega_{1}$ is subject to a possible flux.

where $\partial \Omega_{0}$ is a portion of real boundary (the shoreline) and $\partial \Omega_{1}$ is an artificial boundary through which there might be a flux (see Figure 2). Here $\partial \Omega_{1}$ is included in our derivation because the basin of fluid may be too large to include the whole area in the computation. The user may have some measurements in a coastal area and cannot define $\Omega$ to be the whole ocean. Instead, they will join a segment of the real coastline $\partial \Omega_{0}$ to an artificial boundary $\partial \Omega_{1}$ (called the open boundary) in the ocean to close the domain of interest. If $\partial \Omega=\partial \Omega_{0}$, our method reduces to the first two steps of the three-part algorithm described by Lipphardt et al. [2000].

[13] Equations (8) and (9) are not sufficient to establish boundary conditions for the two functions $\phi$ and $\psi$. Note also that the decomposition given by equation (2) is not unique. Given a particular solution, one can add a divergence-free function to $\phi$ and a zero-vorticity term to $\psi$. This degree of freedom allows us to make the following choice on $\phi$ and $\psi: \bar{u}_{\psi}=\nabla \times \psi \bar{k}$ does not contribute to the flux through the coastline $\partial \Omega_{0}$, i.e., $\bar{n} \cdot \bar{u}_{\psi}=-\bar{t} \cdot \nabla \psi=0$. This is a very natural choice in the sense that if the boundary was completely of type $\partial \Omega_{0}$, we would require $\psi=0$ on the boundary [see, e.g., Eremeev et al., 1995a, 1995b; Lipphardt et al., 2000], which would also imply $\bar{t} \cdot \nabla \psi=0$. As a result, the second term of equation (9) disappears on $\partial \Omega_{0}$ and gives

$$
\bar{n} \cdot \nabla \phi=\bar{u} \cdot \bar{n} \equiv 0 \quad \text { on } \partial \Omega_{0} .
$$

We assume that $\partial \Omega_{0}$ is connected in $\partial \Omega$; that is, there is only one connected segment of shoreline. Figure 2 gives an example of such a geometry. In section 7 , we extend the

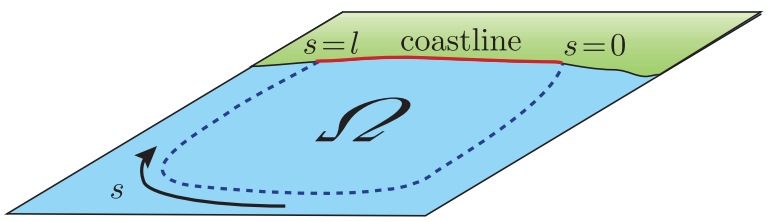

Figure 3. Parameter $s$ is the arc length on the boundary and is defined in such a way that the open boundary runs from $s=0$ to $s=l$. The remainder of the boundary $(s>l)$ is the shoreline.

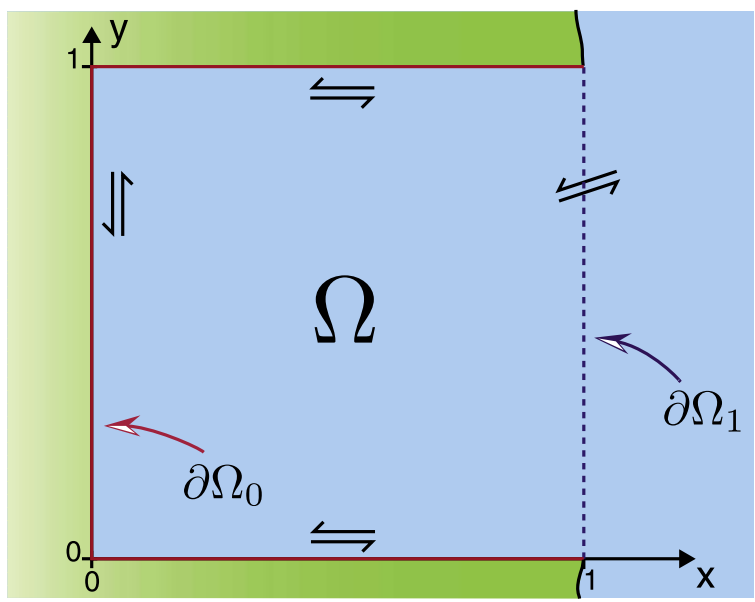

Figure 4. Test domain for OMA. The shoreline corresponds to the left top and bottom edges of the square. Inflow and outflow can occur through the right edge.

results to more complex geometries (see Figure 5 in section 7 , for example). Since we have $\bar{t} \cdot \nabla \psi=0$ on the segment $\partial \Omega_{0}$ and $\psi$ can be added any arbitrary constant function (under the assumption that $\partial \Omega_{0}$ is a connected set), we can assume $\psi=0$ on $\partial \Omega_{0}$. The equations for $\phi$ and $\psi$ then become

$$
\begin{cases}\Delta \phi=\nabla \cdot \bar{u} & \text { in } \Omega, \\ \bar{n} \cdot \nabla \phi=0 & \text { on } \partial \Omega_{0}, \\ \bar{n} \cdot \nabla \phi=g_{\phi}(s) & \text { on } \partial \Omega_{1}\end{cases}
$$

and

$$
\begin{cases}\Delta \psi=-\bar{k} \cdot(\nabla \times \bar{u}) & \text { in } \Omega, \\ \psi=0 & \text { on } \partial \Omega_{0}, \\ \psi=g_{\psi}(s) & \text { on } \partial \Omega_{1},\end{cases}
$$

where $g_{\phi}(s)$ and $g_{\psi}(s)$ are two unknown $L^{2}\left(\partial \Omega_{1}\right)$ functions of the arc length $s$ on the boundary $\partial \Omega_{1}$ that depend on the velocity field. We can also write the equations for $\psi$ and $\phi$ in the following way:

$$
\begin{cases}\Delta \phi=\nabla \cdot \bar{u} & \text { in } \Omega, \\ \bar{n} \cdot \nabla \phi=g_{\phi}(s) & \text { on } \partial \Omega\end{cases}
$$

and

$$
\begin{cases}\Delta \psi=-\bar{k} \cdot(\nabla \times \bar{u}) & \text { in } \Omega, \\ \psi=g_{\psi}(s) & \text { on } \partial \Omega,\end{cases}
$$

where we implicitly mean that $g_{\phi}$ and $g_{\psi}$ vanish on $\partial \Omega_{0}$.

\section{Boundary Modes}

[14] We define the boundary modes to be those obtained by solving the singular but consistent Neumann problem,

$$
\begin{cases}\Delta \phi^{b}=\int_{\partial \Omega} g_{\phi}(s) d s & \text { in } \Omega, \\ \bar{n} \cdot \nabla \phi^{b}=g_{\phi}(s) & \text { on } \partial \Omega .\end{cases}
$$


We assume that a basis $\left\{g_{i}\right\}$ has been chosen for the set $\mathcal{F}$ of scalar $L^{2}$ functions on the boundary with support included in $\partial \Omega_{1}$. Any function $g_{\phi}$ can be written as a linear combination of the basis functions $g_{i}$. Let us define $\phi_{i}^{b}$ by

$$
\begin{cases}\Delta \phi_{i}^{b}=\int_{\partial \Omega} g_{i}(s) d s & \text { in } \Omega, \\ \bar{n} \cdot \nabla \phi_{i}^{b}=g_{i}(s) & \text { on } \partial \Omega .\end{cases}
$$

Notice that if $\phi_{i}^{b}$ is a solution of equation (17), any function $\phi_{i}^{b}+K$, where $K$ is a constant, is also a solution. As a result, we can add the condition

$$
\iint_{\Omega} \phi_{i}^{b} d A=0
$$

and the solutions $\phi_{i}^{b}$ of equation (17) are unique. Suppose that $\phi_{i}^{b}$ and $\phi_{i}^{\prime b}$ are two solutions of equation (17) for the same $g_{i}$. Their difference $\delta=\phi_{i}^{\prime b}-\phi_{i}^{b}$ must satisfy

$$
\begin{cases}\Delta \delta=0 \quad \text { in } \Omega \\ \bar{n} \cdot \nabla \delta=0 & \text { on } \partial \Omega .\end{cases}
$$

It is standard that equations (18) and (19) give $\delta=0$, and so $\phi_{i}^{b}$ is unique.

[15] In sections 7 and 9, we illustrate the method, with a discrete Fourier basis of the function defined on the open boundary,

$$
\left\{g_{i}(s)\right\}=\left\{1, \ldots, \sin \left(\frac{i \pi}{l} s\right), \cos \left(\frac{i \pi}{l} s\right), \ldots\right\}
$$

where $l$ is the length of the open boundary and we assumed that $s>l$ corresponds to the shoreline (see Figure 3 ). This is a natural choice in the sense that these boundary modes satisfy the one-dimensional modal equation

$$
\Delta g_{i}=-\lambda_{i}^{g} g_{i}
$$

Other bases, such as numerical bump functions, can also be used for the discrete set of functions $\left\{\phi_{i}^{b}\right\}$; see Chu et al. [2003] for other examples. In the following sections, we assume that a choice (Fourier basis or other) has been made.

\section{Interior Modes}

[16] The interior eigenmodes, by definition, satisfy the following equations:

$$
\left\{\begin{array} { l l } 
{ \Delta \phi _ { i } = \lambda _ { i } ^ { \phi } \phi _ { i } , } & { \text { in } \Omega , } \\
{ \overline { n } \cdot \nabla \phi _ { i } = 0 , } & { \text { on } \partial \Omega , }
\end{array} \quad \left\{\begin{array}{ll}
\Delta \psi_{i}=\lambda_{i}^{\psi} \psi_{i} & \text { in } \Omega, \\
\psi_{i}=0 & \text { on } \partial \Omega,
\end{array}\right.\right.
$$

where we implicitly assume that the solutions $\phi_{i}$ and $\psi_{i}$ are normalized using

$$
\left\|\phi_{i}\right\|=\left\|\psi_{i}\right\|=1
$$

According to Evans [1998], the modes $\left\{\phi_{i}\right\}$ and $\left\{\psi_{i}\right\}$ form a basis of, respectively, $W^{1}(\Omega)$ and $W_{0}^{1}(\Omega)$. As a result, the associated vector fields $\left\{\nabla \phi_{i}\right\}$ and $\left\{\nabla \times \psi_{i} \bar{k}\right\}$ span the set of all velocities $\bar{u}$ with both components in $L^{2}(\Omega)$ and satisfying the boundary condition $\left.\bar{n} \cdot \bar{u}\right|_{\partial \Omega}=0$ on both the coastline and the open boundary.

[17] Notice that the eigenvalues $\lambda_{i}^{\phi}$ and $\lambda_{i}^{\psi}$ are necessarily negative. Integration by parts gives

$$
\left(\nabla \phi_{i}, \nabla \phi_{i}\right)=\oint_{\partial \Omega} \phi_{i} \bar{n} \cdot \nabla \phi_{i} d s-\left(\phi_{i}, \Delta \phi_{i}\right)=-\left(\phi_{i}, \Delta \phi_{i}\right),
$$

because $\bar{n} \cdot \nabla \phi_{i}=0$ on $\partial \Omega$, and

$$
\left(\nabla \psi_{i}, \nabla \psi_{i}\right)=\oint_{\partial \Omega} \psi_{i} \bar{n} \cdot \nabla \psi_{i} d s-\left(\psi_{i}, \Delta \psi_{i}\right)=-\left(\psi_{i}, \Delta \psi_{i}\right)
$$

because $\psi_{i}=0$ on $\partial \Omega$. This gives

$$
0 \leq\left\|\nabla \phi_{i}\right\|^{2}=-\left(\phi_{i}, \Delta \phi_{i}\right)=-\lambda_{i}^{\phi}\left\|\phi_{i}\right\|^{2}=-\lambda_{i}^{\phi},
$$

and

$$
0 \leq\left\|\nabla \psi_{i}\right\|^{2}=-\left(\psi_{i}, \Delta \psi_{i}\right)=-\lambda_{i}^{\psi}\left\|\psi_{i}\right\|^{2}=-\lambda_{i}^{\psi} .
$$

[18] Hence the energies of the interior eigenmodes are proportional to the absolute value of their eigenvalue

$$
E^{\phi_{i}}=\frac{1}{2} \rho\left\|\nabla \phi_{i}\right\|^{2}=\frac{1}{2} \rho\left|\lambda_{i}^{\phi}\right|
$$

For practical purposes, we assume that the eigenvectors are ordered with respect to the absolute value of their eigenvalue, i.e.,

$$
\left|\lambda_{i}^{\phi}\right| \leq\left|\lambda_{i+1}^{\phi}\right| \quad \text { for all } i
$$

and

$$
\left|\lambda_{i}^{\psi}\right| \leq\left|\lambda_{i+1}^{\psi}\right| \quad \text { for all } i
$$

[19] Now we estimate the characteristic length scale of each mode. As a first approximation, we ignore the exact shape of the mode and note that the only relevant quantities are $\lambda$, the energy $E$, and the characteristic length scale $d$. According to equation (28), energy and eigenvalue are not independent quantities, so we apply Buckingham's $\Pi$-theorem [Buckingham, 1914; Curtis et al., 1982] to $\lambda$ $\left(\left[L^{-2}\right]\right)$ and $d([L])$ to get

$$
d^{2} \lambda=K
$$

Notice that the last relationship holds for any acceptable couple $(d, \lambda)$, and hence for each eigenmode with the same constant $K$. For the first mode, we have

$$
K=d_{1}^{2} \lambda_{1}=D^{2} \lambda_{1},
$$


where we assume that the characteristic length of the domain $D$ is identical to the characteristic length of the first mode. The approximate characteristic length for each mode is then given by

$$
d_{i}=D \sqrt{\frac{\lambda_{1}}{\lambda_{i}}}
$$

\section{Complete Basis}

[20] The nowcast velocity is given by a function $\bar{u}$ on $\Omega$ such that each component belongs to $L^{2}(\Omega)$ and $\bar{u}$ satisfies the boundary condition $\bar{n} \cdot \bar{u}=0$ on $\partial \Omega_{0}$. Assume that we have solved the boundary modes using equation (17) in $W^{1}(\Omega)$ and then define the normal flow on the boundary by the scalar function

$$
g(s)=\left.\bar{n} \cdot \bar{u}\right|_{\partial \Omega} .
$$

Since $\left\{g_{i}\right\}$ is a complete basis of $L^{2}[0, l]$, we can expand $g$ as a linear combination of these vectors and write

$$
g=\sum_{i=1}^{\infty} \alpha_{i}^{b} g_{i}
$$

Define

$$
\bar{u}^{H}=\bar{u}-\sum_{i=1}^{\infty} \alpha_{i}^{b} \nabla \phi_{i}^{b}
$$

and notice that

$$
\begin{aligned}
\left.\bar{n} \cdot \bar{u}^{H}\right|_{\partial \Omega} & =\left.\bar{n} \cdot \bar{u}\right|_{\partial \Omega}-\left.\sum_{i=1}^{\infty} \alpha_{i}^{b} \bar{n} \cdot \nabla \phi_{i}^{b}\right|_{\partial \Omega} \\
& =g(s)-\sum_{i=1}^{\infty} \alpha_{i}^{b} g_{i}(s) \\
& =0 .
\end{aligned}
$$

[21] In other words, $\bar{u}^{H}$ satisfies the homogeneous boundary conditions and each of its components is an $L^{2}(\Omega)$ function. As a result, $\bar{u}^{H}$ can be written as a unique sequence of interior modes,

$$
\bar{u}^{H}=\sum_{i=1}^{\infty} \alpha_{i}^{\psi} \nabla \times \psi_{i} \bar{k}+\sum_{i=1}^{\infty} \alpha_{i}^{\phi} \nabla \phi_{i},
$$

and the velocity nowcast can therefore be written as a unique sequence,

$$
\bar{u}=\sum_{i=1}^{\infty} \alpha_{i}^{\psi} \nabla \times \psi_{i} \bar{k}+\sum_{i=1}^{\infty} \alpha_{i}^{\phi} \nabla \phi_{i}+\sum_{i=1}^{\infty} \alpha_{i}^{b} \nabla \phi_{i}^{b} .
$$

[22] Notice that only the scalar coefficients $\alpha_{i}^{\phi}, \alpha_{i}^{\psi}$, and $\alpha_{i}^{b}$ are functions of time and will change with the data. The velocity modes $\nabla \times \psi_{i} \bar{k}, \nabla \phi_{i}$, and $\nabla \phi_{i}^{b}$ only depend on the geometry. They can be computed once and stored for a particular region. In addition, equation (39) allows for a clear separation of the different length scales involved. Earlier approaches [Lipphardt et al., 2000; Chu et al., 2003] emphasized the importance of the availability of a characteristic length scale for each interior mode (see equation (33)). However, they did not have access to the characteristic length scale of the flow on the open boundary. The OMA nowcast given by equation (39) allows for a uniform filtering by removing (interior and boundary) modes under a prescribed threshold.

\subsection{Incompressible Nowcast}

[23] When the flow is incompressible, the nowcast is most efficiently obtained by adding this extra constraint directly in equation (39). We have

$$
\nabla \cdot \bar{u}=\sum_{i=1}^{\infty} \alpha_{i}^{\phi} \lambda_{i}^{\phi} \phi_{i}+\sum_{i=0}^{\infty} \alpha_{i}^{b} \int_{\partial \Omega} g_{i}(s) d s=0 .
$$

Notice that equation (40) can be seen as a linear combination of the functions $\phi_{i}$ and the constant functions $\int_{\partial \Omega} g_{i}(s) d s$. It is clear from equation (22) that none of the eigenfunctions $\phi_{i}$ can be constant over the domain $\Omega$. If it was the case, we would have

$$
\phi^{i}=\frac{1}{\lambda_{i}^{\phi}} \Delta \phi_{i}=0
$$

and $\phi_{i}$ would be the trivial solution. As a result, each $\phi_{i}$ is linearly independent from the constant functions $\int \partial \Omega g_{i}(s) d s$ in equation (40) and the divergence of the velocity field vanishes if and only if

$$
\alpha_{i}^{\phi}=0, \quad \text { for all } i
$$

and

$$
\sum_{i=0}^{\infty} \alpha_{i}^{b} \int_{\partial \Omega} g_{i}(s) d s=0
$$

As a result, the nowcast of an incompressible flow can be written as a unique sequence

$$
\bar{u}=\sum_{i=1}^{\infty} \alpha_{i}^{\psi} \nabla \times \psi_{i} \bar{k}+\sum_{i=1}^{\infty} \alpha_{i}^{b} \nabla \phi_{i}^{b}
$$

with an additional constraint on the coefficients of the boundary modes given by equation (43).

\subsection{Incompressible Nowcast With Fourier Basis}

[24] If we use a Fourier basis for the boundary functions, only one function $g_{i}$ (corresponding to $\cos \frac{\pi s}{l}$ ) has a nonzero integral over the boundary. As a result, equation (43) reduces to

$$
\alpha_{1}^{b}=0
$$

and the nowcast is simply given by omitting this term in equation (44),

$$
\bar{u}=\sum_{i=1}^{\infty} \alpha_{i}^{\psi} \nabla \times \psi_{i} \bar{k}+\sum_{i=2}^{\infty} \alpha_{i}^{b} \nabla \phi_{i}^{b} .
$$

\section{Example: The Unit Square}

[25] We consider the domain depicted in Figure 4 and given by

$$
\Omega=[0,1] \times[0,1],
$$


where

$$
\begin{aligned}
\partial \Omega_{0}= & \{0 \leq x<1 \text { and } y=0\} \\
& \cup\{0 \leq x<1 \text { and } y=1\} \\
& \cup\{0 \leq y \leq 1 \text { and } x=0\}
\end{aligned}
$$

is the solid boundary (coastline) and

$$
\partial \Omega_{1}=\{0 \leq y \leq 1 \text { and } x=1\}
$$

allows transport. The interior modes are given by

$$
\left\{\begin{array}{l}
\psi_{k, l}=\sin (k \pi x) \sin (l \pi y) \\
\phi_{k, l}=\cos (k \pi x) \cos (l \pi y),
\end{array}\right.
$$

and correspond to the eigenvalues

$$
\lambda_{k, l}^{\phi}=\lambda_{k, l}^{\psi}=-\pi^{2}\left(k^{2}+l^{2}\right) .
$$

[26] The associated velocity modes are given by

$$
\left\{\begin{array}{c}
\bar{u}_{k, l}^{\psi}=\nabla \times \psi_{k, l} \bar{k}=\pi\left(\begin{array}{c}
k \sin (k \pi x) \cos (l \pi y) \\
-l \cos (k \pi x) \sin (l \pi y)
\end{array}\right), \\
\bar{u}_{k, l}^{\phi}=\nabla \phi_{k, l}=-\pi\left(\begin{array}{c}
k \sin (k \pi x) \cos (l \pi y) \\
l \cos (k \pi x) \sin (l \pi y)
\end{array}\right) .
\end{array}\right.
$$

[27] Using a Fourier basis of the functions defined on $\partial \Omega_{1}$, we can compute the boundary modes. We illustrate the process by computing the modes corresponding to

$$
g_{k}(s)=g_{k}(y)=\pi \cos (k \pi y) .
$$

One can verify that the unique solution to equation (17) is

$$
\phi_{k}^{b}=\frac{e^{k \pi x}+e^{-k \pi x}}{e^{k \pi}-e^{-k \pi}} \cos (k \pi y),
$$

and the corresponding velocity fields are

$$
\bar{u}_{k}^{b}=\nabla \phi_{k}^{b}=\frac{1}{k}\left(\begin{array}{c}
\frac{e^{k \pi x}-e^{-k \pi x}}{e^{k \pi}-e^{-k \pi}} \cos (k \pi y) \\
-\frac{e^{k \pi x}+e^{-k \pi x}}{e^{k \pi}-e^{-k \pi}} \sin (k \pi y)
\end{array}\right) .
$$

[28] For $g_{k}(y)=\sin (k \pi y)$, we could not find an analytical solution. A detailed algorithm to compute numerical OMA modes can be found in section 9 .

\section{Fully Open Boundaries and Islands}

[29] The definition of the OMA modes accepts many different geometries of the boundary. Equation (17) gives the definition of the boundary modes. In this definition, a)

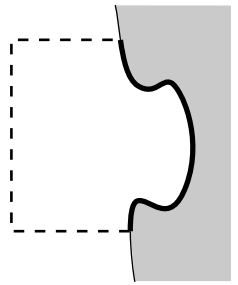

c)

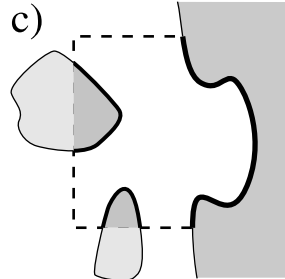

e)

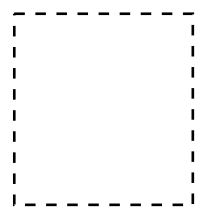

b)

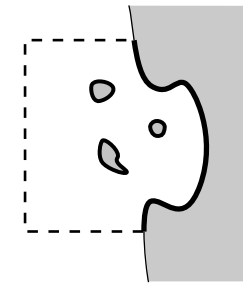

d)

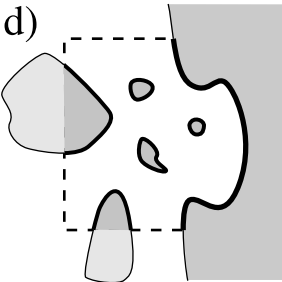

f)

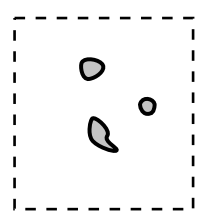

Figure 5. Possible domains of application for OMA. Thick solid lines are segments of $\partial \Omega_{0}$. Dashed lines are segments of $\partial \Omega_{1}$. (a, b) Basic OMA domain with or without islands. (c, d) Disconnected open boundary (resulting from the presence of islands that are not completely contained in $\Omega$ ). (e, f) Completely open boundary (i.e., $\partial \Omega_{0}=\emptyset$ ).

the functions $g_{i}$ are the elements of a basis of the function defined on $\partial \Omega$, whose support is included in $\partial \Omega_{1}$. In the settings above, there was only one non-empty segment of coastline and only one segment of open boundary. In this case, the functions $g_{i}$ are a basis of the set of all scalar functions defined on $\partial \Omega_{1} \otimes$ the zero function on $\partial \Omega_{0}$. Chu et al. [2003] show how to treat domains with multiple islands (and a single open boundary). The same remark applies to this work, since the boundaries of the islands are appended to $\partial \Omega_{0}$ and there is still only one connected segment of open-boundary $\partial \Omega_{1}$ (see Figures $5 \mathrm{a}$ and $5 \mathrm{~b}$ ). In addition, OMA allows many more different configurations. The two major examples are completely open domains and disconnected open boundaries.

\subsection{Open Domains}

[30] In some cases, the outermost boundary may be completely open. This is the case for nowcast in the open ocean (where none or some islands may be present inside the domain, as depicted in Figures 5e and 5f. In this case, the open-boundary $\partial \Omega_{1}$ is a closed curve. The functions $g_{i}$ are still a basis of the scalar function on the open boundary, but $\partial \Omega_{1}$ is topologically equivalent to the circle, $S^{1}$. According to our definition, values of $g_{i}$ are now defined on $S^{1}$; hence a Cartesian representation constrains them to periodic functions $g_{i}(0)=g_{i}(l)$.

\subsection{Disconnected Open Boundaries}

[31] Another important case occurs when one or several islands inside the domain are not completely contained inside $\Omega$. In this case, the islands separate the open 
boundary into multiple segments (see Figures $5 \mathrm{c}$ and $5 \mathrm{~d}$ ). Unlike other methods, OMA is particularly well suited for this type of situation. If $\partial \Omega_{1}$ is split between several disjoint segments $\partial \Omega_{1}^{j}$, a basis of the scalar functions on $\partial \Omega_{1}$ can still be computed and used to determine the boundary modes. In this case, the $g_{i}$ span the set

$$
\mathcal{F}_{\partial \Omega_{1}}=\mathcal{F}_{\partial \Omega_{1}^{1}} \otimes \mathcal{F}_{\partial \Omega_{1}^{2}} \otimes \cdots \mathcal{F}_{\partial \Omega_{1}^{q}} \otimes \mathcal{F}_{\partial \Omega_{0}},
$$

where $\mathcal{F}_{\partial \Omega_{1} !}$ is a basis of the scalar functions on the $j$ th segment of open-boundary $\partial \Omega_{1}^{j}$.

[32] Although careful design of the geometry to avoid disconnected boundaries is sometimes possible, OMA's ability to treat almost any possible geometry is an important improvement over earlier methods. The smallest domain providing the fastest nowcast will not necessarily satisfy more restrictive conditions such as the multiply connected open domain condition of Chu et al. [2003].

\section{Extrapolation, Interpolation, and Filtering}

[33] Equation (39) can be rewritten in the more compact form,

$$
\bar{u}=\sum_{n=1}^{\infty} \alpha_{n} \bar{u}_{n},
$$

where $\bar{u}_{n}$ represents any of the linearly independent modes (boundary, incompressible, or irrotational). Since the sequence of modes is infinite, the optimal coefficients $\alpha_{n}$ cannot be determined with a finite number of measurements. In addition, the smallest details are obtained for high eigenvalues, and using a finite sequence as an approximation to equation (57) allows one to filter the data and keep significant details only. The nowcast at a particular time is given by

$$
\bar{u}^{\prime}=\sum_{n=1}^{N} \alpha_{n} \bar{u}_{n},
$$

where $N$ is the total number of modes (irrotational, incompressible, and boundary) and $\bar{u}_{n}$ represent any of these linearly independent modes. The modes have been ordered with respect to their characteristic lengths (i.e., their eigenvalues), so the $N$ first modes contain the largest features of the flow. According to equation (33), if $\bar{u}_{N}$ is an interior mode, the finite sequence typically ignores details smaller than $D \sqrt{\lambda_{1}} / \sqrt{\lambda_{N}}$. This allows for a length-scale based criteria for filtering the data. Using only $N$ modes means that the coefficients corresponding to the other modes are arbitrarily set to zero; that is, $\bar{u}^{\prime}$ is the projection of the real velocity $\bar{u}$ in a finite-dimensional subspace of the space of all available $L^{2} \times L^{2}(\Omega)$ velocities that are tangent to $\partial \Omega_{0}$. To compute those coefficients, we need at least $N$ measurements. In this paper, the coefficients $\alpha_{n}$ will be the solution of a least squares minimization problem where the function to minimize $\zeta\left(\alpha_{n}\right)$ is a function of the error $\bar{u}^{\prime}-\bar{u}$. Notice that a least square minimization problem may not be the optimal choice. Chu et al. [2003] propose several other norms and compare the resulting nowcasts.

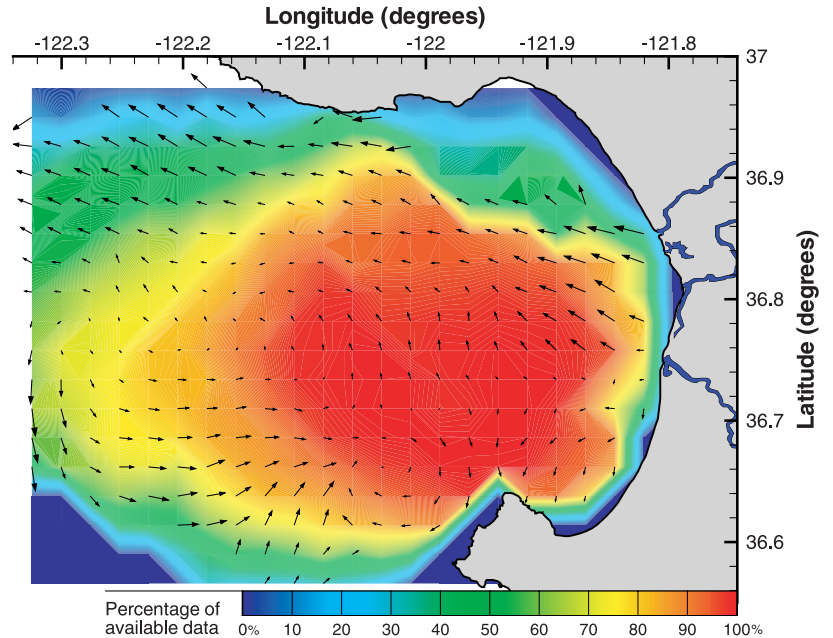

Figure 6. Black vectors show a footprint of the HF radar data in Monterey Bay on 1 August 0700 GMT. Also shown on this picture are the level sets of the percentage of available data during the month of August 2000. Regions in red have data almost all the time. Data is unavailable most of the time in the blue regions.

[34] Assuming that we have $k$ measurements $\bar{u}_{q}^{\text {mes }}$ at the position $\bar{x}_{q}=\left(x_{q}, y_{q}\right)$, the value of $\bar{u}$ is only known at $k$ points, where

$$
\bar{u}\left(\bar{x}_{q}\right)=\bar{u}_{q}^{\text {mes }}
$$

we define the error as

$$
\zeta=\sqrt{\sum_{q}\left\|\bar{u}^{\prime}\left(\bar{x}_{q}\right)-\bar{u}\left(\bar{x}_{q}\right)\right\|^{2}} ;
$$

that is,

$$
\zeta=\sqrt{\sum_{q}\left\|\sum_{n} \alpha_{n} \bar{u}_{n}\left(\bar{x}_{q}\right)-\bar{u}_{q}^{\operatorname{mes}}\right\|^{2}} .
$$

Using the $L^{2}$ norm in equation (61) gives a least squares minimization problem. The set of projection coefficients $\alpha_{i}$ minimizes the error when

$$
\frac{\partial \zeta}{\partial \alpha_{j}}=0, \forall j
$$

that is,

$$
\sum_{n} \alpha_{n} \sum_{q} \bar{u}_{n}\left(\bar{x}_{q}\right) \cdot \bar{u}_{j}\left(\bar{x}_{q}\right)=\sum_{q} \bar{u}_{q}^{\mathrm{mes}} \cdot \bar{u}_{j}\left(\bar{x}_{q}\right), \forall j .
$$

Equation (63) is a linear system of $N$ equations with $N$ unknowns $\alpha_{i}$, and its solution gives the set of optimal coefficients determining the filtered nowcast. 




Figure 7. Adaptive mesh at the end of the computation of the second incompressible mode $\psi_{2}$. Figure 8 shows an enlargement of the region highlighted by the red box.

[35] Notice that solving equation (63) or computing the projection of the data using other norms [Chu et al., 2003] is a simple and costless operation. For real-time applications, the velocity modes can be stored, and OMA only requires solving a linear system of $N$ equations $(N$ is of the order of 10 to 100$)$ at each time step. This is a valuable improvement over the method presented by Chu et al. [2003], where all the modes have to be recomputed at each time step.

\section{Application to Monterey Bay}

[36] This application uses high-frequency (HF) radar technology [Paduan and Rosenfeld, 1996; Paduan and Cook, 1997; Prandle and Ryder, 1985; Goldstein and Zebker, 1987; Georges et al., 1996], which is now able to resolve time-dependent Eulerian flow features in surface currents along coastlines. Such an HF radar installation has been operating in Monterey Bay (California coastline) since

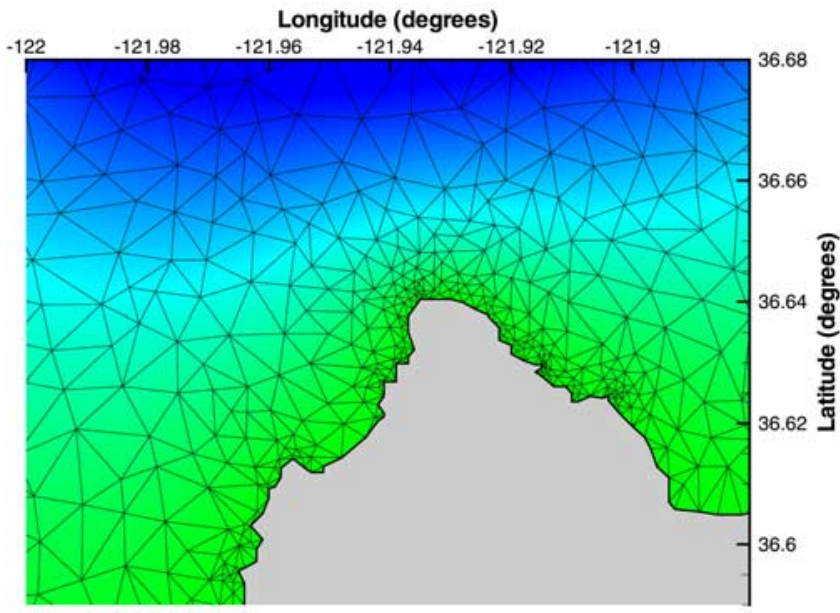

Figure 8. Unstructured mesh for the computation of $\psi_{2}$ near Point Piños, the southernmost point of the bay (see red box on Figure 7), where the flow separates between the bay and the Pacific Ocean.

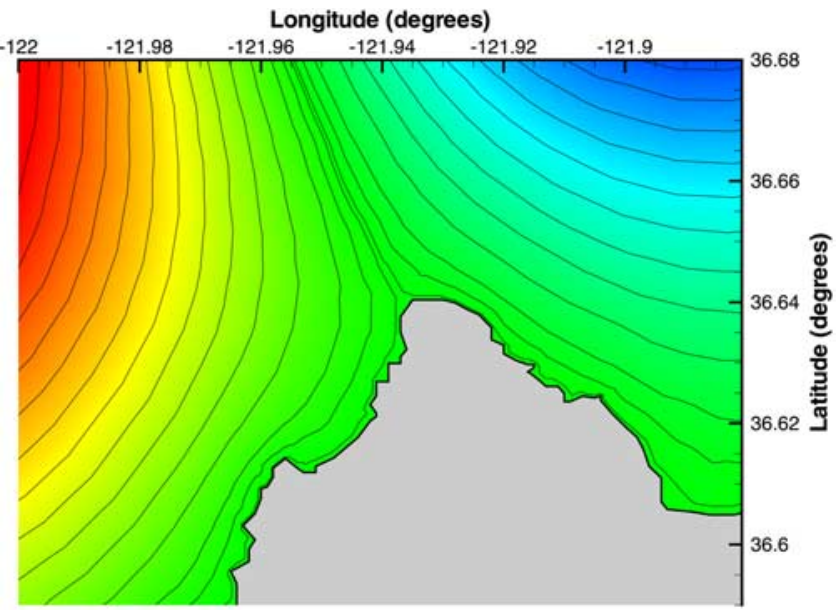

Figure 9. Streamlines of the fourth incompressible interior boundary mode $\psi_{4}$ in the Point Piños area. The streamline matches the boundary at a high level of precision.

1994 [Paduan and Rosenfeld, 1996; Paduan and Cook, 1997]. In our study, we use data from this installation, acquired by the three HF radar antenna systems near Monterey Bay. The observational data were collected in August 2000, binned every hour on a uniform grid with $1 \mathrm{~km}$ by $1 \mathrm{~km}$ intervals.

[37] An example of an HF radar footprint of Monterey Bay at 0700 GMT, 1 August 2000, is shown in Figure 6. Also shown on Figure 6 are the level sets of the percentage of available data in the bay for the month of August 2000. There are usually many gaps in the data at each time, especially near the open boundary and in the uppermost region of the bay. The reason for this distribution is the range of the radar system. The performance decreases with the distance. Also, some regions do not have surface waves high enough to scatter the signal, and the radars cannot determine the velocity vectors. Our objective is to build a method that filters, interpolates, and extrapolates such incomplete data sets.

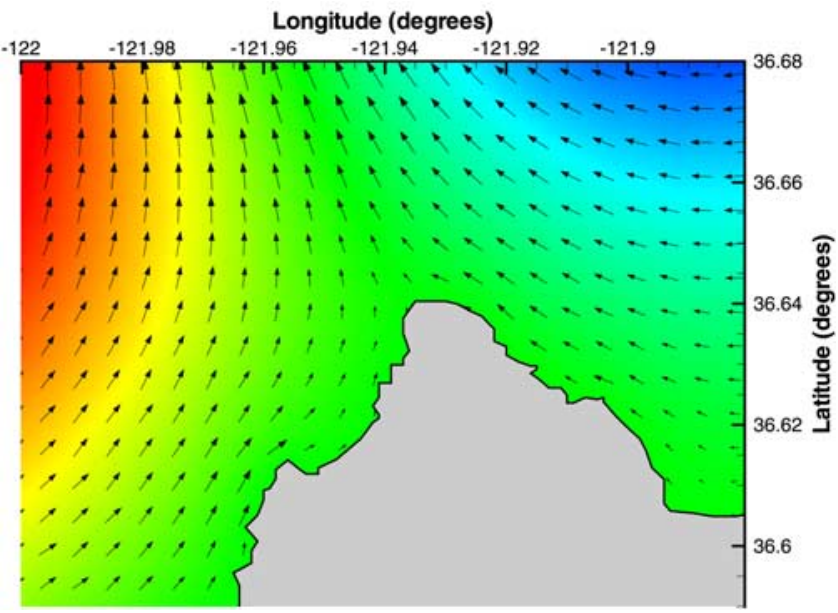

Figure 10. Velocity field $\bar{u}_{4}{ }^{\psi}$ corresponding to the fourth incompressible interior mode $\psi_{4}$ of Figure 9 in the Point Piños area. 

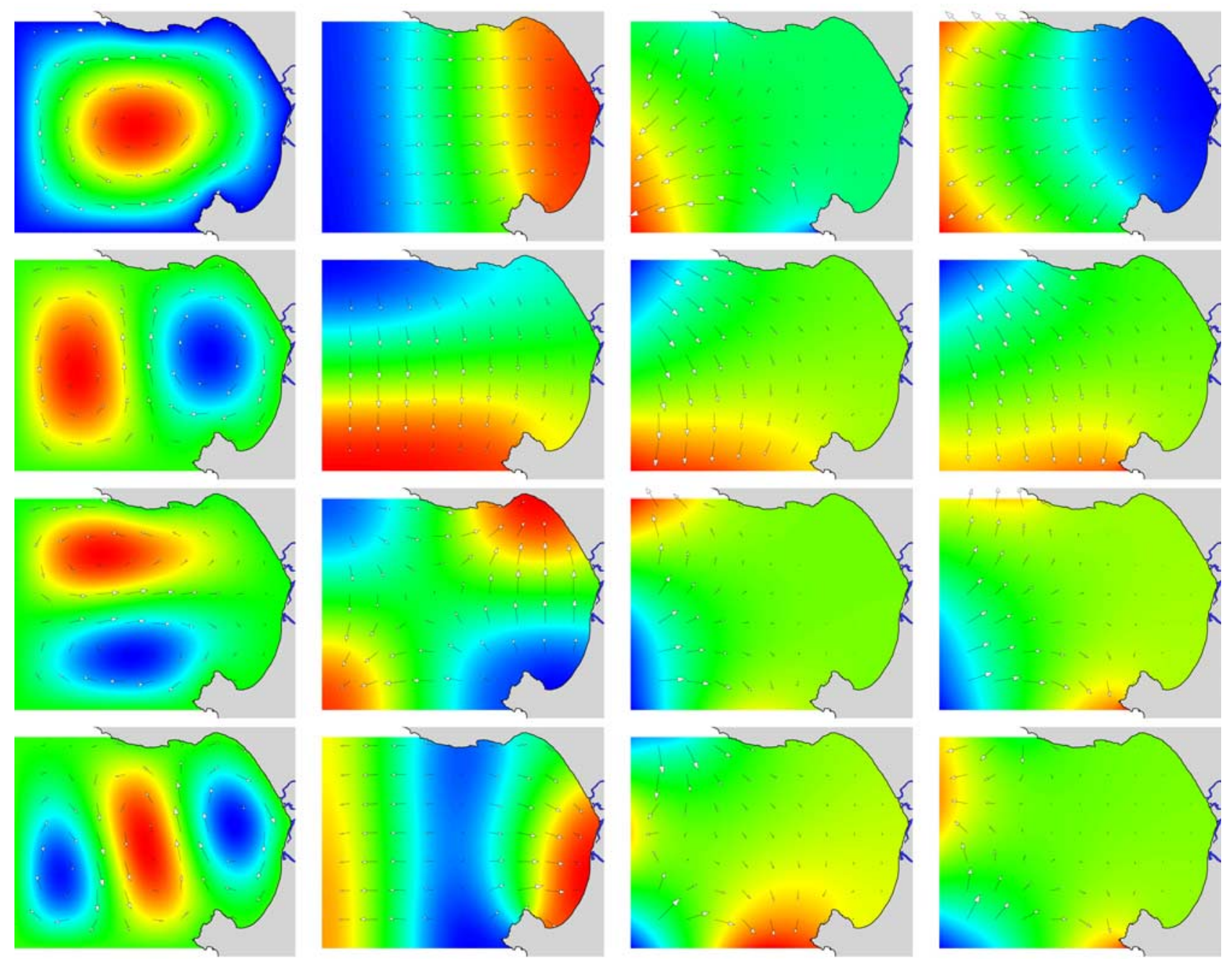

Figure 11. First OMA modes computed for Monterey Bay. The first column shows the incompressible modes $\psi_{1}, \psi_{2}, \psi_{3}$, and $\psi_{4}$. The second column shows the irrotational modes $\phi_{1}, \phi_{2}, \phi_{3}$, and $\phi_{4}$. The last two columns contain boundary modes for, respectively, $g_{i=1 \rightarrow 4}=\sin \frac{i \pi s}{L}$ and $g_{i=0 \rightarrow 3}(s)=\cos \frac{i \pi s}{L}$.

[38] A high-precision version of the shoreline for our domain was extracted on a topological map and is visible on Figure 6. We use a numerical software package called PLTMG (http://www.scicomp.ucsd.edu/reb) to solve the mode equations (equations (22) and (17)). Unlike other elliptic PDE solvers, PLTMG renders the eigenfunctions on an unstructured triangular grid with a general boundary. Figure 7 shows a particular instance of the adaptive mesh used to compute the incompressible interior mode $\psi_{2}$. The use of an unstructured mesh is necessary for applications such as the integration of particles or the computation of Lagrangian structures near a complicated shoreline. Inadequate representation of the shoreline or discrepancies between the computed velocity field and the free slip boundary condition often result in particles erroneously crossing the shoreline. Figure 8 shows the unstructured mesh in a magnified region centered on Point Piños, the southernmost part of the bay featured on Figure 7. The flow typically separates between the bay and the ocean at Point Piños. This region is a dynamical hot spot in our Lagrangian studies (C. Coulliette et al., Optimal pollution release in Monterey Bay based on nonlinear analysis of coastal radar data, submitted to Journal of Environmental Science and Technology, 2004) (hereinafter referred to as Coulliette et al., submitted manuscript, 2004), and our work requires very refined velocities in this area. Figures 9 and 10 show the streamlines of the mode $\psi_{4}$ and the corresponding velocity field $\bar{u}_{4}^{\psi}$ in the Point Piños area. Only unstructured meshes provide streamlines so compatible with such a complicated coastline. In the work of Lipphardt et al. [2000] and Chu et al. [2003] the coastline is approximated by a staircase boundary (i.e., a polygon composed entirely of segments that are normal, or at right angles). The staircase boundary is a much less accurate representation of a coastline geometry than the unstructured triangulation that we used. One might try to use an extremely fine staircase boundary. However, this is computationally intractable because it would require making the entire domain an equally fine mesh. In this case, not only would the computational time be prohibitive, but round-off error becomes a limiting factor.

[39] In addition, the unstructured mesh is adapted to the solution computed. Advanced algorithms detailed by Bank [1994] allow dynamic modifications of the mesh to keep a 


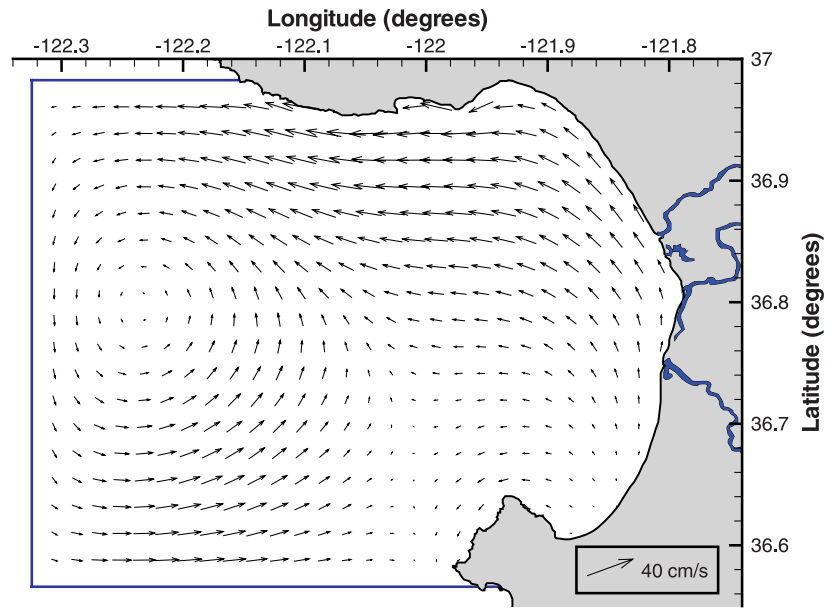

level sets of the irrotational interior modes (second column) and boundary modes (third and fourth columns).

[41] Figure 12 shows two nowcasts realized with OMA. The upper nowcast uses only four incompressible modes and four irrotational modes. It cannot produce any flow normal to the boundary. It is similar to the nowcasts obtained after the first two steps of the algorithm described by Lipphardt et al. [2000]. The lower nowcast uses eight additional boundary modes and is able to generate normal flow on the open boundary (interaction with the Pacific Ocean). The error between the HF radar data (red arrows on Figure 13) and the nowcast, which does not use boundary modes (black arrows on Figure 13), can be extremely large near the open boundary. Figure 13 also reveals that


Figure 12. Effect of the boundary modes on the nowcast computed for 1 August 2000 at 0700 GMT. Both nowcasts use four incompressible modes and four irrotational modes. The nowcast in the top panel does not use any boundary and cannot produce any flow through the open boundary. The nowcast in the bottom panel uses eight boundary modes.

constant numerical error in calculating the mode shapes. The error across the domain and boundary is held constant by varying the size of the triangles. On Figure 7, smaller triangles are used close to the boundary to match the highresolution curve. In addition, PLTMG has increased the number of triangles for this mode in selected regions, such as the corners of the open boundary or the northern and southern part of the line dividing the two gyres. This design provides a numerical error that is constant for the whole domain, thus reducing the number of elements for a given error threshold. In comparison, a normal grid with constantsize elements results in a numerical error that varies in magnitude substantially across the domain and boundary [see, e.g., Lipphardt et al., 2000; Chu et al., 2003]. The unstructured triangulation is more efficient and results in a much higher accuracy to computational time ratio.

[40] The first few modes computed for Monterey Bay are represented in Figure 11. The corresponding velocity modes are tangent to the streamlines of the incompressible interior modes (first column of Figure 11) and orthogonal to the

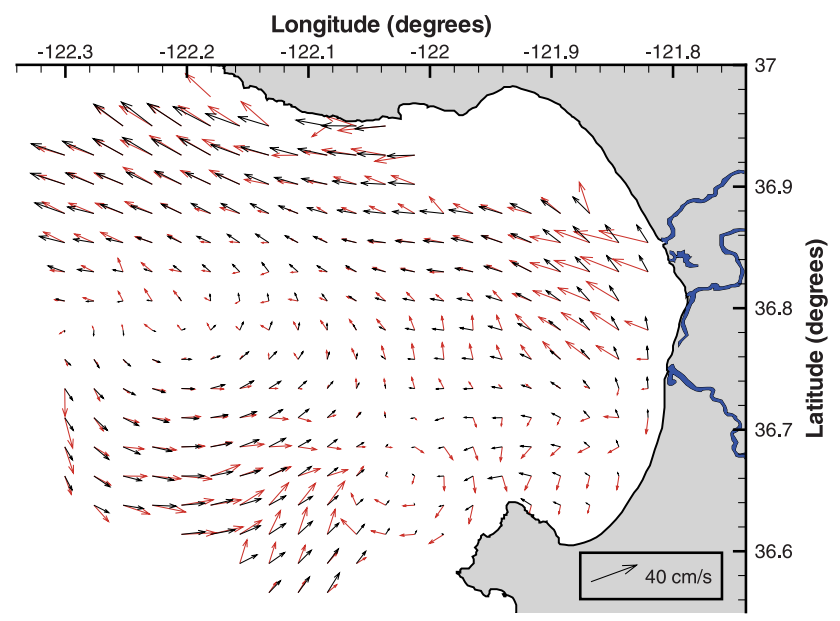

Figure 13. Effect of the boundary modes on the nowcast computed for 1 August 2000 at 0700 GMT. On both images, the red vectors represent the available HF radar data for that time. The black arrows are the velocity at the same points evaluated by the OMA nowcast. The nowcast in the top panel uses only four incompressible modes and four irrotational modes. Since the upper nowcast does not use any boundary mode, the error between the HF radar data and the nowcast near the open boundary is large. The nowcast in the bottom panel uses eight additional boundary modes and is able to reduce the error between experimental data and extrapolated velocity. 

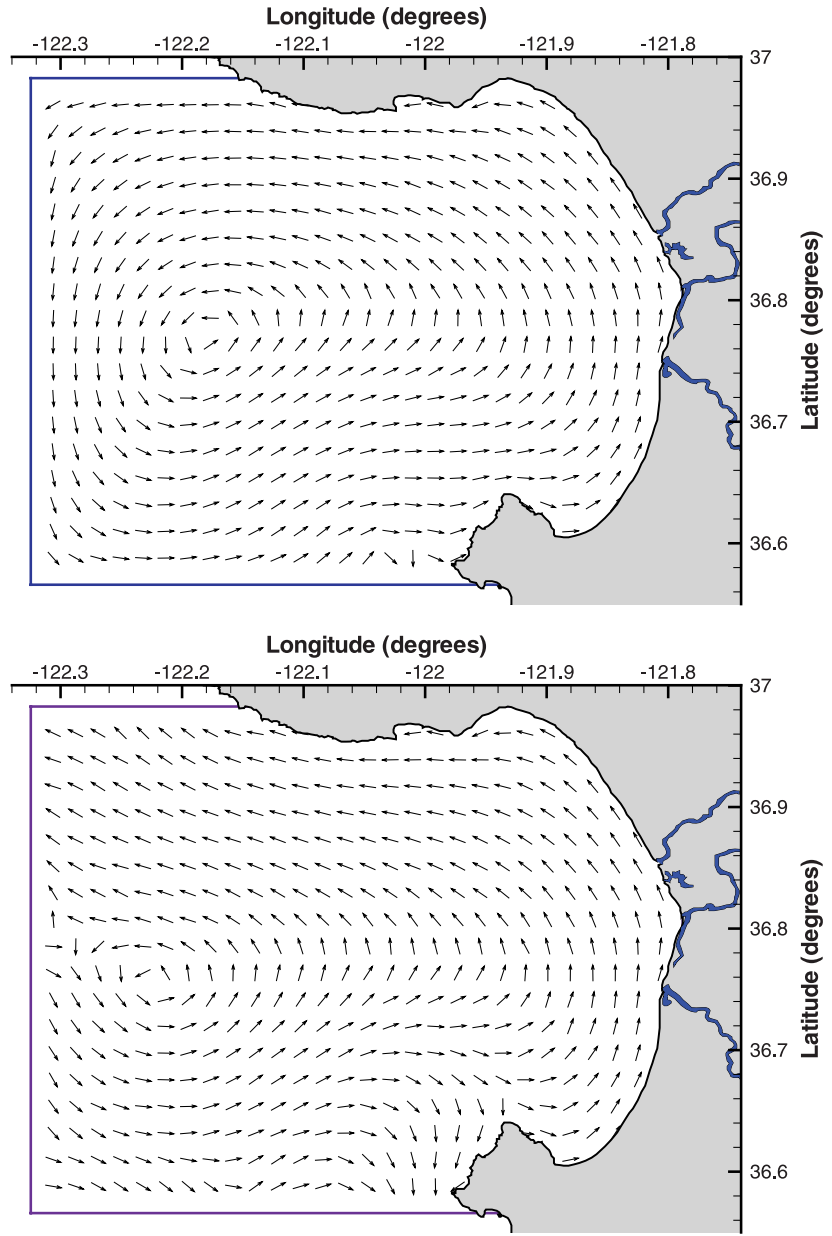

Figure 14. Effect of the boundary modes on the nowcast computed for 17 August 20001200 GMT. The top panel uses four incompressible modes and four irrotational modes. The bottom panel uses four additional boundary modes. The velocity vectors have been normalized on each panel to highlight the differences between the dynamical features. Only the OMA nowcast (bottom panel) is able to reproduce the separation point on the shoreline (near Point Piños) that was visible in the HF radar data. This example shows that certain important dynamical features can be wiped by removing the boundary modes.

including eight boundary modes in the nowcast decreases the error significantly near the open boundary.

[42] One might wonder if the effect of the boundary modes is only a minor correction of the nowcast near the open boundary. If this is the case, computations inside the bay (far away from the open boundary) may not be affected by the boundary modes. Figure 14 shows the nowcast on 17 August 2000 at 1200 GMT. The upper nowcast does not use boundary modes. These are included only in the lower nowcast of Figure 14.

[43] One can notice that the two nowcasts are dynamically different, even inside the bay, far away from the open boundary. For the closed-boundary nowcast (Figure 14, top), there is no separation point on the coastline. The flow spirals around the Bay. However, the open-boundary modes of the full OMA nowcast (Figure 14, bottom) captured the separation point near Point Piños.

\section{Conclusion}

[44] We presented a practical method to interpolate, extrapolate, and filter experimental Eulerian data. This is the first modal analysis that includes a sequence of boundary modes. As a consequence, the modeler does not need to speculate on the open-boundary flux. Previous approaches like that of Lipphardt et al. [2000] require the use of a larger model or an extrapolation of the data to determine the flux across the open boundary. As shown by Chu et al. [2003], the modes computed and the nowcasts are very sensitive to numerical and experimental errors in the determination of an a priori flux through the open boundary. In addition, earlier methods do not produce time-independent modes and require the computation of some or all of the modes at each time step. In contrast, OMA adapts the flow near the boundary with the available data through equation (63). If the normal flow is known near the boundary, OMA uses this information and provides nowcasts similar to the adapted three-step algorithm of Lipphardt et al. [2000]. If, at some time, data are available only in the middle of the domain, far away from the boundary, OMA naturally projects the data on the boundary modes and finds the boundary flow that best fits the data. The space spanned by the OMA modes is much larger than the spaces spanned by Lipphardt et al. [2000] or Chu et al. [2003]. However, the numerical complexity of the nowcasting problem is much smaller when using autonomous OMA modes. These can be stored and read from a file during an experiment and do not need to be computed in a real-time setting.

[45] There are many applications of such methods. Our group is primarily concerned with the interpolation, filtering, and extrapolation of Eulerian velocities. Other examples include Eulerian prediction, which was discussed by Chu et al. [2003]. Recently, the motion of a vector containing the projection coefficients of the nowcast velocity was used by Lekien and Leonard [2004] to determine dynamical regimes (such as upwelling or double-gyre patterns) in experimental data.

[46] Other mode generation techniques are available today. In particular, statistical modes have been introduced in the context of fluid flows in the work by Lumley [1970]. An extensive application of statistical modes for compressible flows is given by Rowley et al. [2004]. A major difference between OMA and statistical modes is that OMA modes do not depend on the data. The statistical modes are adapted to the data distribution, and fewer modes are usually necessary to obtain the same precision. It is our impression that an adapted basis of statistical modes will always give better nowcasts with fewer modes than modal analysis. Compared to statistical modes, the strength of modal analysis is its potential to generate autonomous modes. The approach described by Lipphardt et al. [2000] seems to develop modes that are independent of the observed field, but in fact, a zero-order mode solution is required at every time step. While this is computationally expensive, this is much more efficient than the method of Chu et al. [2003] that requires the computation of each mode at each time step. In contrast, OMA modes have the 
advantage of being completely independent of the field observed. The modes are computed once for a given boundary geometry and can be stored for future use, which makes them attractive for long-term applications with enormous amounts of data and very useful for real-time computations, such as current or even Lagrangian forecasting as described by Lekien et al. [2003], Coulliette et al. (submitted manuscript, 2004), and F. Lekien et al. (Pollution release tied to invariant manifolds: A case study for the coast of Florida, submitted to Physica D, 2004).

\section{Notation}

$\bar{x}$ position (longitude, latitude).

$\bar{u}(\bar{x}) \quad$ velocity at point $\bar{x}$.

$\bar{u}^{\prime}(\bar{x})$ filtered nowcast velocity at point $\bar{x}$.

$\psi$ stream function.

$\psi_{i}$ incompressible interior mode.

$\bar{u}_{i}^{\psi}$ incompressible velocity mode derived from $\psi_{i}$.

$\phi$ relative vorticity.

$\phi_{i}$ irrotational interior mode.

$\bar{u}_{i}^{\phi} \quad$ irrotational velocity mode derived from $\phi_{i}$.

$\phi_{i}^{b} \quad$ boundary mode.

$\bar{u}_{i}^{b} \quad$ boundary velocity mode derived from $\phi_{i}^{b}$.

$\Omega$ domain covered.

$\partial \Omega$ boundary of $\Omega$.

$\partial \Omega_{0}$ coastline.

$\partial \Omega_{1}$ open boundary.

$\bar{k}$ unit vector pointing upward.

$\bar{n}$ unit vector normal to the boundary pointing outward.

$\bar{t} \quad$ tangent vector $\bar{t}=\bar{n} \times \bar{k}$.

[47] Acknowledgments. The authors are grateful to the Office of Naval Research for their support (grant N00014-01-1-0208 and the AOSNii project N00014-02-1-0826), particularly program managers Manuel Fiedero, Reza Malek-Madani, and Wen Masters. The authors also thank Bruce Lipphardt and Michael Toner for enlightening discussions and enriching ideas. The high-frequency radar data were collected in Monterey Bay by the Naval Postgraduate School, and the authors are grateful to Jeffrey Paduan and Michael Cook for processing and sharing available velocity fields.

\section{References}

Bank, R. E. (1994), PLTMG: A Software Package for Solving Elliptic Partial Differential Equations: User's Guide, 7.0, Soc. of Ind. and Appl. Math., Philadelphia, Pa

Buckingham, E. (1914), On physically similar systems: Illustrations of the use of dimensional equations, Phys. Rev. 4, 345-376.

Chu, P. C., L. M. Ivanov, T. P. Korzhova, T. M. Margolina, and V. Melnichenko (2003), Analysis of sparse and noisy ocean current data using flow decomposition, J. Atmos. Oceanic Technol., 20, 478491.

Coulliette, C., and S. Wiggins (2001), Intergyre transport in a wind-driven, quasi-geostrophic double gyre: An application of lobe dynamics, Nonlinear Proc. Geophys., 8, 69-94.

Curtis, W. D., J. D. Logan, and W. A. Parker (1982), Dimensional analysis and the pi theorem, Linear Algebra Appl., 47, 117-126.

Eiseman, P. R., and A. P. Stone (1973), Hodge decomposition theorem, Notices Am. Math. Soc., 20(1), A169.

Eremeev, V. N., L. M. Ivanov, and A. D. Kirwan (1992a), Reconstruction of oceanic flow characteristics from quasi-Lagrangian data: 1 . Approach and mathematical methods, J. Geophys. Res., 97(C6), 9733-9742.

Eremeev, V. N., L. M. Ivanov, A. D. Kirwan, O. V. Melnichenko, S. V. Kochergin, and R. R. Stanichnaya (1992b), Reconstruction of oceanic flow characteristics from quasi-Lagrangian data: 2. Characteristics of the large-scale circulation in the Black Sea, J. Geophys. Res., 97(C6), 97439753.

Eremeev, V. N., L. M. Ivanov, A. D. Kirwan, and T. M. Margolina (1995a), Amount of cs-137 and cs-134 radionuclides in the Black Sea produced by the Chernobyl accident, J. Environ. Radioact., 27(1), 49-63.

Eremeev, V. N., L. M. Ivanov, A. D. Kirwan, and T. M. Margolina (1995b), Analysis of cesium pollution in the Black Sea by regularization methods, Mar. Pollut. Bull., 30(7), 460-462.

Evans, L. C. (1998), Partial differential equations, in Graduate Studies in Mathematics, vol. 19, Am. Math. Soc., Providence, R. I.

Georges, T. M., J. A. Harlan, and R. A. Lematta (1996), Large-scale mapping of ocean surface currents with dual over-the-horizon radars, Nature, $379,434-436$.

Goldstein, R. M., and H. A. Zebker (1987), Interferometric radar measurement of ocean surface currents, Nature, 328, 707-709.

Lekien, F., and N. Leonard (2004), Dynamically consistent Lagrangian coherent structures, AIP Conf. Proc., in press.

Lekien, F., C. Coulliette, and J. Marsden (2003), Lagrangian structures in very high-frequency radar data and optimal pollution timing, AIP Conf. Proc., 676, 162-168.

Lipphardt, B. L., Jr., A. D. Kirwan Jr., C. E. Grosch, J. K. Lewis, and J. D. Paduan (2000), Blending HF radar and model velocities in Monterey Bay through normal mode analysis, J. Geophys. Res., 105(C2), 3425-3450.

Lumley, J. L. (1970), Stochastic Tools in Turbulence, Am. Inst. of Phys., College Park, Md.

Paduan, J. D., and M. S. Cook (1997), Mapping surface currents in Monterey Bay with codar-type HR radar, Oceanography, 10, 49-52.

Paduan, J. D., and L. K. Rosenfeld (1996), Remotely sensed surface currents in Monterey Bay from shore-based HF radar (codar), J. Geophys. Res., 101, 20,669-20,686.

Prandle, D., and D. K. Ryder (1985), Measurement of surface currents in Liverpool Bay by high-frequency radar, Nature, 315, 128-131.

Rowley, C. W., T. Colonius, and R. M. Murray (2004), Model reduction for compressible flows using POD and Galerkin projection, Physica D, $189(1-2), 115-129$.

R. Bank, Department of Mathematics, University of California at San Diego, La Jolla, CA 92093-0112, USA. (rbank@ucsd.edu)

C. Coulliette and J. Marsden, Control and Dynamical Systems, 107-81, California Institute of Technology, Pasadena, CA 91125, USA. (chadc@cds.caltech.edu; marsden@cds.caltech.edu)

F. Lekien, Department of Mechanical and Aerospace Engineering, Princeton University, EQuad J220, Princeton, NJ 08544, USA. (lekien@ princeton.edu) 